\title{
A new measurement of the cosmic X-ray background
}

\author{
A. Moretti ${ }^{1}$, C. Pagani ${ }^{2}$, G. Cusumano ${ }^{3}$, S. Campana ${ }^{1}$, M. Perri ${ }^{4}$, A. Abbey ${ }^{5}$, M. Ajello ${ }^{6,7}$, A. P. Beardmore ${ }^{5}$, \\ D. Burrows ${ }^{2}$, G. Chincarini ${ }^{1,8}$, O. Godet $^{5}$, C. Guidorzi ${ }^{1,8}$, J. E. Hill ${ }^{9}, 10$, J. Kennea ${ }^{2}$, J. Nousek ${ }^{2}$, \\ J. P. Osborne ${ }^{5}$, and G. Tagliaferri ${ }^{1}$
}

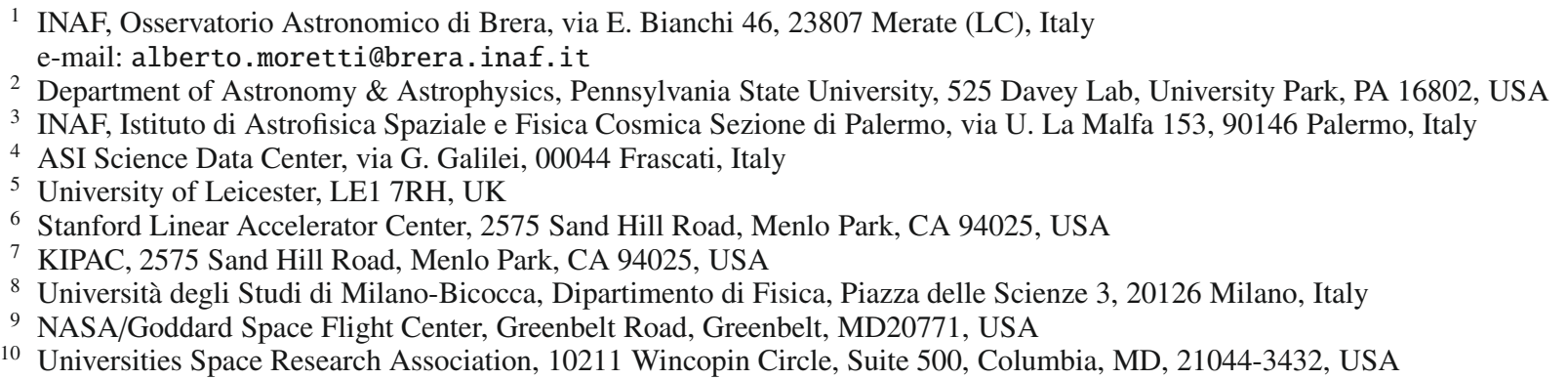

Received 21 October 2008 / Accepted 31 October 2008

\section{ABSTRACT}

\begin{abstract}
Aims. We present a new measurement of the cosmic X-ray background (CXRB) in the $1.5-7 \mathrm{keV}$ energy band, performed by exploiting the Swift X-ray telescope (XRT) data archive. We also present a CXRB spectral model in a wider energy band (1.5-200 keV), obtained by combining these data with the recently published Swift-BAT measurement.

Methods. From the XRT archive we collect a complete sample of 126 high Galactic latitude gamma-ray burst (GRB) follow-up observations. This provides a total exposure of $7.5 \mathrm{Ms}$ and a sky-coverage of $\sim 7$ square degrees which represents a serendipitous survey, well suited for a direct measurement of the CXRB in the 1.5-10 keV interval. Our work is based on a complete characterization of the instrumental background and an accurate measurement of the stray-light contamination and vignetting calibration.

Results. We find that the CXRB spectrum in the $1.5-7 \mathrm{keV}$ energy band can be equally well fitted by a single power-law with photon index $\Gamma=1.47 \pm 0.07$ or a single power-law with photon index $\Gamma=1.41 \pm 0.06$ and an exponential roll-off at $41 \mathrm{keV}$. The measured flux in the $2-10 \mathrm{keV}$ energy band is $2.18 \pm 0.13 \times 10^{-11} \mathrm{erg} \mathrm{cm}^{-2} \mathrm{~s}^{-1} \mathrm{deg}^{-2}$ in the $2-10 \mathrm{keV}$ band. Combining Swift-XRT with Swift-BAT $(15-200 \mathrm{keV})$ we find that, in the $1.5-200 \mathrm{keV}$ band, the CXRB spectrum can be well described by two smoothly-joined power laws with the energy break at $29.0 \pm 0.5 \mathrm{keV}$ corresponding to a $v F_{v}$ peak located at $22.4 \pm 0.4 \mathrm{keV}$.

Conclusions. Taking advantage of both the Swift high energy instruments (XRT and BAT), we produce an analytical description of the CXRB spectrum over a wide (1.5-200 keV) energy band. This model is marginally consistent with the HEAO1 measurement ( $\sim 10 \%$ higher) at energies higher than $20 \mathrm{keV}$, while it is significantly $(30 \%)$ higher at low energies $(2-10 \mathrm{keV})$.
\end{abstract}

Key words. X-rays: diffuse background - surveys - instrumentation: miscellaneous

\section{Introduction}

The cosmic X-ray background (CXRB) is usually defined as the integrated emission of all the extragalactic sources in the X-ray energy band $(\sim 2-100 \mathrm{keV})$. The name background comes directly from the first X-ray astronomical observation (Giacconi et al. 1962), when an apparently diffuse background was observed together with the first extra-solar X-ray source (Sco X-1). The CXRB spectral properties, flux and isotropy were accurately (10\%) measured over a wide energy band by the A2 and A4 experiments on board the high energy astronomical observatory 1 (HEAO1) satellite. The analytical model produced by Gruber et al. (1999), combining A2 and A4 observations with higher energy data has been considered as a reference for many years (G99 model hereafter). Much effort has been spent to quantify the fraction of CXRB emission due to unresolved point sources. As predicted by Giacconi \& Zamorani (1987) a combination of large and deep surveys performed by focusing telescopes in the soft part of the X-ray spectrum $(<10) \mathrm{keV}$ has succeeded in resolving almost the entire (80-90\%) CXRB, the resolved fraction decreasing at higher energies (Moretti et al. 2003; Worsley et al. 2005). The point sources producing the resolved fraction of CXRB in the $2-10 \mathrm{keV}$ band have been found to be mostly AGN with a small contributions from galaxy clusters and starburst galaxies (Hornschemeier et al. 2000; Bauer et al. 2004; Brandt \& Hasinger 2005; Tozzi et al. 2006). Furthermore, a highly anisotropic diffuse component is present at energies lower than $1 \mathrm{keV}$ (Sołtan 2007). This is contributed by the Local Bubble, the Galactic halo (Galeazzi et al. 2007) and the intergalactic medium (Cen \& Ostriker 1999), while at higher energies, and high Galactic latitude the diffuse component is negligible.

There is a general consensus on the sources from which the CXRB originates, and the background paradox can be considered solved (Setti \& Woltjer 1989); nevertheless, the spectrum of the X-ray integrated emission is still very important in the study of the statistical properties of those sources that are too faint to be detected individually by currently operating telescopes, as highly absorbed AGNs and very high red shift quasars. The extrapolation of the AGN observed spectra (unabsorbed and 
Table 1. A compilation of CXRB flux measurements in the soft energy band sorted by year of publication, compared with the G99 model.

\begin{tabular}{lcc}
\hline \hline Instrument & $\begin{array}{c}\text { Flux 2-10 keV } \\
{\left[10^{-11} \mathrm{erg} \mathrm{cm}^{-2} \mathrm{~s}^{-1} \mathrm{deg}^{-2}\right]}\end{array}$ & Reference \\
\hline HEAO1 & $1.65 \pm 0.17$ & Gruber et al. (1999) \\
Rockets & $2.20 \pm 0.20$ & McCammon et al. (1983) \\
ASCA-SIS & $1.92 \pm 0.09$ & Gendreau et al. (1995) \\
SAX-MECS & $2.35 \pm 0.10$ & Vecchi et al. (1999) \\
ASCA-GIS & $1.94 \pm 0.20$ & Kushino et al. (2002) \\
RXTE-PCA & $1.64 \pm 0.05^{*}$ & Revnivtsev et al. (2003) \\
XMM-Newton & $2.24 \pm 0.16$ & De Luca \& Molendi (2004) \\
HEAO1-A2 & $1.66 \pm 0.08^{*}$ & Revnivtsev et al. (2005) \\
Chandra & $2.19 \pm 0.26$ & Hickox \& Markevitch (2006) \\
\hline
\end{tabular}

* The original values are $1.91 \pm 0.06$ and $1.96 \pm 0.10$ respectively. We correct them, according to the values reported in Table 3 of Revnivtsev et al. (2005) to account for the cross-calibration with XMM-Newton.

Compton-thin) to the region of the CXRB peak ( $30 \mathrm{keV})$ can account for $\sim 75 \%$ of the peak (Gilli et al. 2007). Compton-thick AGNs are thought to be responsible for the remaining fraction (Treister \& Urry 2005; Gilli et al. 2007; Ballantyne \& Papovich 2007). Given that even the most recent AGN surveys in the hard band, $>10 \mathrm{keV}$, can add only few percent to this number (Sazonov et al. 2007, 2008; Tueller et al. 2008), the CXRB provides a key boundary condition in the determination of the census of the heavily obscured AGNs. Moreover, an accurate measurement of the CXRB spectrum is an important observational constraint in the study of the very high redshift $(z>6)$ AGNs which will remain unresolved even with the next generation of X-ray telescopes (Salvaterra et al. 2007; Rhook \& Haehnelt 2008). Finally, a proper characterization of the CXRB spectrum is also crucial to ensure proper background subtraction in the study of low surface brightness diffuse X-ray emission coming from the outskirts of clusters and groups of galaxies (Gastaldello et al. 2007; Snowden et al. 2008).

Measurements performed in the soft part of the CXRB spectrum with different instruments (see Table 1) yield a scatter which is much larger than the one expected from standard candle flux measurements (Kirsch et al. 2005), meaning that the observed discrepancy cannot be entirely explained by the differences in absolute calibrations of the individual instruments. The large scatter and the poor control on systematic uncertainties in the CXRB measurements led some authors (Ueda et al. 2003; Treister \& Urry 2005) to use the G99 model, re-normalized by a factor of $\sim 30 \%$. The underlying (but not verified) assumption is that the G99 spectrum is correct in shape but with the normalization affected by some calibration problems of the HEAO1 instruments. Worsley et al. $(2005,2006)$ use an even more artificial solution, combining the XMM-Newton CXRB measurement (De Luca \& Molendi 2004) up to $8 \mathrm{keV}$ and the re-normalized G99 at higher energies. On the other hand, recently published measurements, performed by means of wide-field not-focused hard X-ray instruments (SAX-PDS, INTEGRAL-IBIS, SwiftBAT) yield results consistent (10\% level) with the G99 model in the 20-50 keV range (Churazov et al. 2007; Frontera et al. 2007; Ajello et al. 2008), reversing the recent trend that prefers higher intensities (Ueda 2007).

Here we present a new measurement of the CXRB spectrum in the $1.5-7 \mathrm{keV}$ energy band, obtained by the analysis of the archival data of the X-ray telescope (XRT) on board the Swift satellite (Gehrels et al. 2004), a mission dedicated to the study of gamma-ray bursts (GRBs) and their afterglows. XRT uses a Wolter I mirror set, originally designed for the JET-X telescope (Citterio et al. 1994), to focus X-rays (0.2-10 keV) onto a XMM-Newton/EPIC MOS CCD detector (Burrows et al. 2005). GRBs are detected and localized by the Burst Alert Telescope (BAT)(Barthelmy et al. 2005), in 15-300 keV energy band and followed-up at X-ray energies $(0.3-10 \mathrm{keV})$ by the X-ray Telescope. Following-up gamma-ray burst afterglows, the Swift-XRT obtains deep exposures on random positions of the sky, totally uncorrelated with already known bright X-ray sources, providing us with a simple and direct measurement of the CXRB spectrum.

\section{Work strategy}

For each energy $E$ we can consider the signal $S_{\text {tot }}$ registered in a typical high Galactic latitude GRB afterglow follow-up observation, as the sum of 4 factors. These are the GRB signal, the CXRB itself, which is the one we aim to measure, the electronic and particle-induced background (NXB or instrumental background) and the stray-light (SL), i.e. the contamination from sources outside the telescope field of view

$$
S_{\text {tot }}(E)=\operatorname{CXRB}(E) \times f_{\text {vign }}(E)+\mathrm{NXB}(E)+\mathrm{SL}(E)+\mathrm{GRB}(E) .
$$

The GRB afterglow signal can be easily eliminated by filtering data both in space and in time. The CXRB itself is contributed by bright resolved plus faint unresolved sources and it is affected by vignetting $\left(f_{\text {vign }}\right)$. We measure the NXB using two different and independent datasets: a two- day observation performed with the focal plane camera assembly (FPCA) sun-shutter closed (SC) and the data collected in a region of the detector which is not exposed to the sky (NES). We evaluate the third element, the SL contamination using a series of off-axis observations of bright sources. Given the high level of the CXRB isotropy (Shafer \& Fabian 1983; Revnivtsev et al. 2008) we can consider this factor as a fraction of the $\mathrm{CXRB}\left(\mathrm{SL}=f_{\mathrm{SL}} \times \mathrm{CXRB}\right)$ in such a way that:

$\operatorname{CXRB}(E)=\frac{S_{\text {tot }}(E)-\mathrm{NXB}(E)}{f_{\text {vign }}(E)+f_{\mathrm{SL}}(E)}$.

To measure the CXRB spectrum we perform stacked spectral analysis of a large sample made only of GRB follow-up observations. The following four sections are devoted to fully describing the technical details of our work, which allowed us to estimate the terms of Eq. (2) and their uncertainties. In particular, in Sect. 3 we describe the dataset and the reduction procedures; in Sects. 4 and 5 we present how we measure the NXB and SL contamination, respectively. In Sect. 6 we describe how we calculate the vignetting correction and its uncertainty. In Sect. 7 we present the spectral analysis procedure and its results, discussing them in Sect. 8.

Throughout this paper, all errors are quoted at $90 \%$ confidence level, unless otherwise specified. The photon index is denoted as $\Gamma$.

\section{Sample selection and data reduction}

During the first 45 months of operation (Jan. 2005-July 2008), the Swift-XRT observed some 300 GRB afterglows with typical exposure times of $70-100 \mathrm{ks}$ during the $\sim 10$ days following the prompt event. We consider all the long $\left(T_{90}>2 \mathrm{~s}\right)$ GRB follow-up observations with a nominal standard exposure longer than $10 \mathrm{ks}$ and Galactic latitude $|b|>20^{\circ}$. Because we find long term variations of the NXB level we consider only data after January 2006 in such a way that the NXB scatter remains lower 
than $10 \%$ (see Sect. 4$)$. Similar variations ( $\$ 3 \%$ per year) in the NXB level were observed in SAX-LECS-MECS and ASCA-GIS (Perri \& Giommi 2000; Kushino et al. 2002) and were interpreted as due to a gradual drop in the satellite altitude and/or to the cycle of solar activity.

The sample consists of 126 GRB observations from January 2006 to July 2008. For each observation we exclude from our analysis the data collected in the first day (the segment 0 ) in order to exclude the brightest phases of the afterglows. For each observation we consider only the central 200 pixel radius $(7.9$ arcmin) circle, excluding a 30 pixel radius (1.18 arcmin), corresponding to $\sim 95 \%$ of the encircled energy fraction of a point-like source (Moretti et al. 2005) around the GRB position. This corresponds to a nominal field of view (FOV) of 0.054 square degrees. The real observed sky solid angle varies from observation to observation depending on the precise pointing distribution of the observation.

We reduce data using the standard software (HEADAS software, v6.4, CALDB version Dec07) and following the procedures in the instrument user guide ${ }^{1}$. We replace the standard good time interval (GTI) definition, which is tuned for the observations of bright point-like sources, by more restrictive filters. Due to the failure of the thermo-electric cooler power supply, the XRT CCD temperature is subject both to orbital $\left(4{ }^{\circ} \mathrm{C}\right.$ in $5.9 \mathrm{ks})$ and long term $\left(15{ }^{\circ} \mathrm{C}\right.$ on a day time scale) variations, ranging between $-70{ }^{\circ} \mathrm{C}$ and $-47^{\circ} \mathrm{C}$ (Kennea et al. 2005). Dark current and hot pixels are highly temperature dependent and create high instrumental background in the low energy band (0.3-0.7 keV) during observations performed at temperatures higher than $-52{ }^{\circ} \mathrm{C}$ (Pagani et al. 2007; Moretti et al. 2007). Moreover, due to the low orbit of the Swift satellite, a typical target can be observed no more than 1-2 ks on a single orbit. Therefore the data from single object are split in different segments. Occasionally some reflected light from the Earth limb significantly increases the very low energy $(<0.5 \mathrm{keV})$ background at the end or at the beginning of an observation segment. To reduce these effects, we select intervals with CCD temperature $<-55{ }^{\circ} \mathrm{C}$ and elevation angle (i.e. the altitude of the observation direction on the Earth horizon) $>40^{\circ}$, instead of the standard $-47^{\circ} \mathrm{C}$ and $30^{\circ}$, respectively. Moreover, we consider only data from observation segments longer than $300 \mathrm{~s}$ and eliminate the first and the last $100 \mathrm{~s}$ of each orbital segment. After the complete time-filtering procedure, these procedures typically reduce the effective exposure time to $50 \%$ of the standard ones. The total nominal exposure time considered is $7.5 \mathrm{Ms}$ with median value of $40 \mathrm{ks}$ for single observations. The final exposure time distribution of the 126 observations, together with the skycoverage is shown in Fig. 1: the surveyed area is 7 and $1.3 \mathrm{deg}^{2}$ at 10 and $100 \mathrm{ks}$ respectively.

\section{Instrumental and particle induced signal}

To evaluate the instrumental and particle induced background (NXB) we use two different datasets.

First, we use the two day observation performed between 2007-09-04 18:50:00 UT and 2007-09-06 18:42:00 UT when the instrument Sun shutter $(0.38 \mathrm{~mm}$ thickness stainless steel of grade 302) was closed due to an improper slew which brought the XRT to point $\sim 15$ degrees from the Sun. The instrument automatically closed the shutter in front of the camera. For the next two days the usual XRT observations were performed, but with

\footnotetext{
1 http://heasarc.nasa.gov/docs/swift/analysis/\# documentation
}
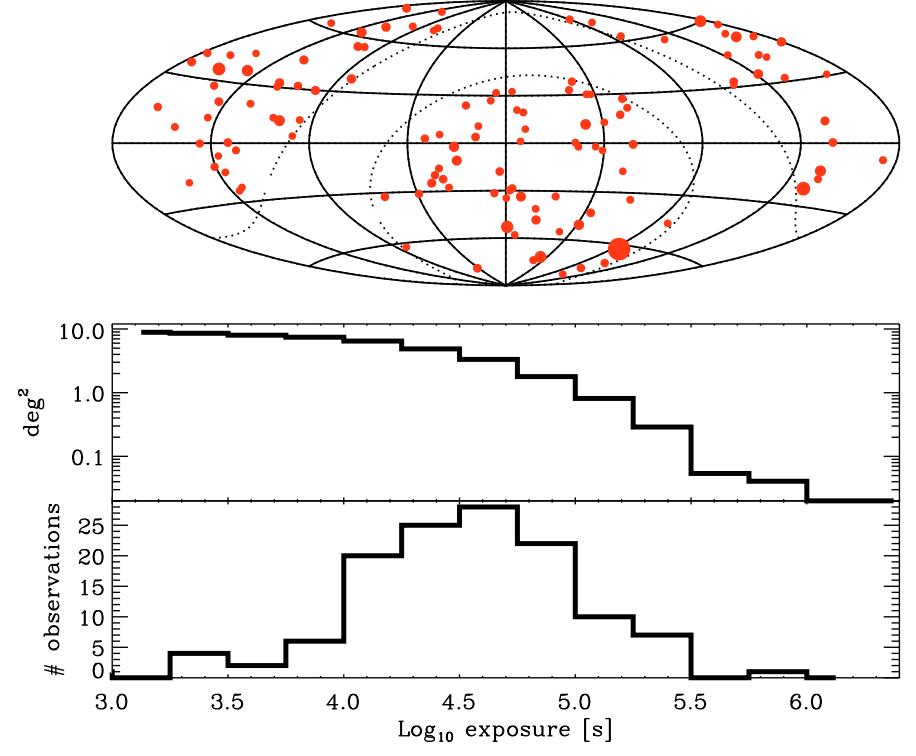

Fig. 1. Upper panel: sky distribution of the 126 observations in Equatorial coordinate, with the Galactic plane exclusion (dotted lines). The size of the points is proportional to the exposure and it is not representative of the observed field size. Middle panel: the cumulative survey sky coverage as a function of (logarithm of) exposure time. Lower panel: distribution of the (logarithm of) nominal exposure time.

the shutter closed. We apply to these data the same reduction and filtering procedures that we apply to the sky data. The final exposure time for the instrumental background with the shutter closed is $67 \mathrm{ks}$ with an average count rate of $1.92 \pm 0.05 \times 10^{-7}$ $\left(7.71 \pm 0.3 \times 10^{-8}\right)$ counts s$^{-1}$ pixel $^{-1}$ in the $0.3-10(1.5-7.0) \mathrm{keV}$ energy band. For the remainder of the paper we will refer to this dataset as shutter closed (SC) dataset.

The second dataset is provided by the data collected in the regions of the detector which are not exposed to the sky (NES). These are four different regions (2507 pixels each) close to the CCD boundary and delimited by the FOV and the corner sources (Fig. 2). The FOV and corner source region definitions are reported in the standard calibration file (CALDB) swxregion20010101v003.fits. In particular the nominal field of view of the telescope is the 300 pixel radius circle centered on the detector pixel $(300,300)$. We conservatively adopted a wider definition of the FOV which is the 323 pixel radius circle centered in the detector pixel $(307,300)$. Then, we define the NES as the parts of the box centered in detector pixel (307, 300), width 436 and height 596 lying outside the conservative $\mathrm{FOV}^{2}$. The signal registered in these regions has been telemetered since June 2008 when the telemetered detector area was increased to $600 \times 600$ pixels. We use all the available data present in the Swift-XRT archive of the photon counting (PC) observations between June-July 2008. This results in a total exposure of 2.4 Ms. The uncertainty in the determination of NXB is one of the main sources of uncertainty of this measurement. For both these datasets we assume that the signal is contributed only by the particle induced and the pure instrumental background. Possible sources of systematic errors are the following. First, the fluorescence from the shutter itself: if the shutter produces some fluorescence lines, our NXB estimate would be systematically higher than the correct value. Second, significant inhomogeneities in the CCD response or in the intrinsic

\footnotetext{
2 In simpler words, according to ds9 syntax, this corresponds to $\operatorname{box}(307,300,436,596,0)-\operatorname{circle}(307,300,323)$.
} 


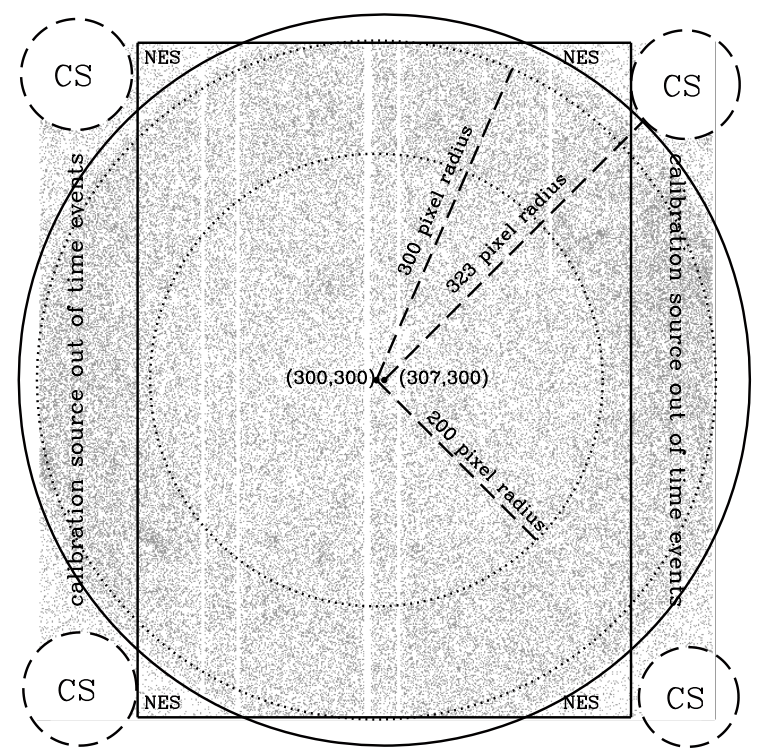

Fig. 2. XRT detector. The inner dotted circle indicates the detector region we consider for the CXRB measurement. The larger dotted circle indicates the nominal field of view. The continuous circle shows the conservative definition of field of view we use to define the NES regions. The continuous box is used to define the NES border and shows the regions contaminated by calibration source (CS) out of time events.

fluorescence background, could bias the NXB measurement in the NES regions. The third error source is the time dependence of the NXB. As already mentioned, in some previous missions, where the NXB background has been estimated by means of observation of the dark Earth, a time dependence on time scale of years has been observed.

To check our data for these systematic errors, we first verify consistency between the two datasets, SC and NES. Comparing the two datasets we find that the NXB in the $1.5-7 \mathrm{keV}$ band displays a gradient in the vertical direction of the CCD, with the bottom regions being $30 \%$ lower than the top regions. This trend is very well described by a linear fit with SC and NES datasets being highly consistent (Fig. 3). The consistency of the two datasets give us good confidence that the first two sources of systematics are negligible for our purposes. We do not have a direct way to monitor the NXB time dependence during all the observations (NES data started to be telemetered only in June 2008) over the entire energy band. Nevertheless we can use the data in the 7-8 keV interval, where the CXRB signal is low and the detected signal is dominated by the $\mathrm{Ni} \mathrm{K}_{\alpha}$ line at $7.48 \mathrm{keV}$ produced by the fluorescence of the telescope material. This is uniformly distributed over the detector area with a typical count rate of $3.8 \times 10^{-8}$ count s$^{-1}$ pixel $^{-1}$. We compare the $\mathrm{Ni}$ line observed in the NES regions with the one observed during the SC and sky observations. To minimize the statistical error we consider the 56 observations longer than $40 \mathrm{ks}$. We model the data in the $6.8-8.2 \mathrm{keV}$ energy band by means of a Gaussian plus a straight line. We find that the Ni line flux decreases slightly with mission time (correlation at $2.5 \sigma$ confidence) producing scatter of $\sim 10 \%$ with respect to the average (Fig. 4). We note that the line flux registered in SC and NES data is perfectly consistent with the average of the observed fluxes. For the purpose of the stacked spectral analysis we account for this uncertainty in the final error budget as explained below in Sect. 7 .

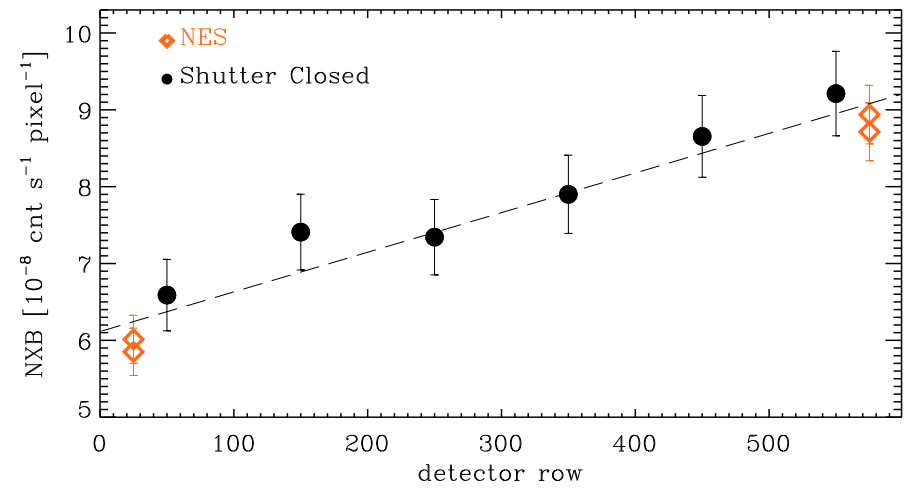

Fig. 3. NXB count rate (per pixel) in the $1.5-7 \mathrm{keV}$ energy band as measured by SC and NES datasets. The linear gradient in the CCD vertical direction is evident.

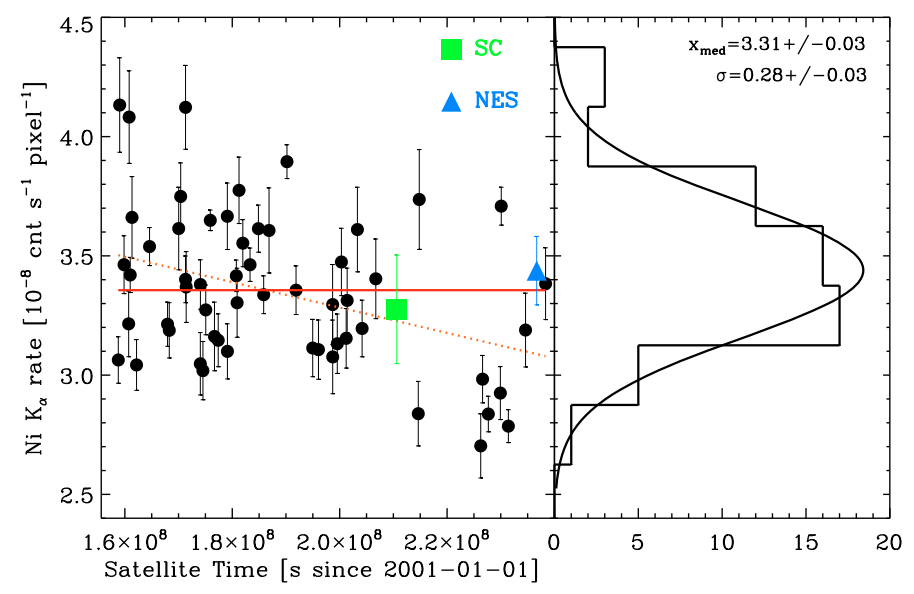

Fig. 4. The instrumental $\mathrm{Ni}_{\alpha}$ line flux registered in the 56 observations with exposure times longer than $40 \mathrm{ks}$. The continuous line is the average, while the dotted line is the best linear fit. The total scatter of these measurements with respect of the average is at the level of $10 \%(1 \sigma)$, as shown in the right panel of the figure.

\section{Stray-light}

The other main component of the non-cosmic background is the SL. Ray-tracing simulations indicate that this is produced by photons coming from sources that are outside the telescope FOV at distance between 20 and 100 arcmin from the optical axis of the telescope, whereas the FOV of the telescope (mirror + detector) has a radius of $\sim 12$ arcmin. A fraction of the photons produced by these sources reach the detector after only one reflection on the mirror or even directly, passing through the mirror shells without any interaction. Some X-ray telescopes mount baffles on top of the mirrors to prevent such a contamination. This is not the case for XRT, for which the SL is a significant fraction of the diffuse radiation registered on the CCD.

In order to evaluate the level of contamination in XRT images, we take advantage of the many Crab Nebula calibration observations. Then a series of observations at 7 different off-axis angles, ranging from 15 to 90 arcmin were performed. We compare them with the on-axis calibration observation. We calculate the fraction of the source flux present on the central 200 pixel radius circle of the detector as the ratio between the flux observed at each distance from the optical axis and the on-axis flux. Given the large off-axis angles the dimensions of the Crab nebula are negligible for our purposes. The results of this analysis are shown in Fig. 5. We split our analysis in several different energy bands, finding no significant variation as function of energy up 

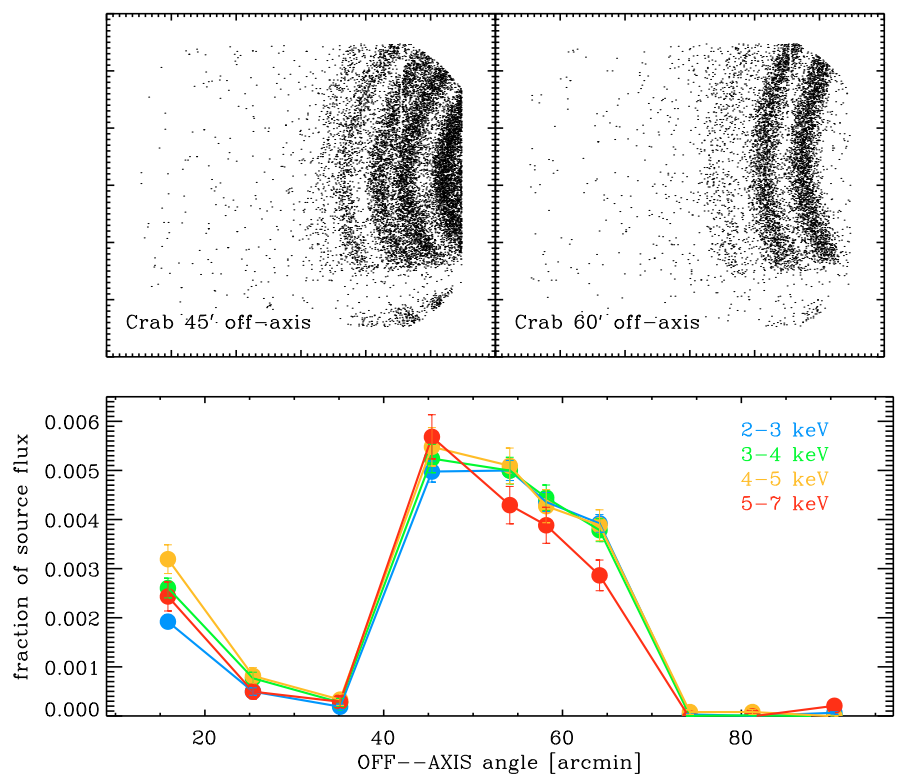

Fig. 5. Upper panel: off-axis observations of the Crab nebula, used to calibrate the SL contamination. Lower panel: ratio between SL and onaxis flux from the Crab at different off-axis angles.

to $5 \mathrm{keV}$. These observations clearly show that XRT images are contaminated by the emission of sources outside the field of view up to $\sim 70$ arcmin. Due to the isotropy of the CXRB we can calculate the expected contamination as the surface integral of the (measured) relative flux produced by the Crab observed at different off-axis angles. The result of the integration in the $1.5-7 \mathrm{keV}$ band is the CXRB fraction $f_{\mathrm{SL}}=0.268 \pm 0.015$ (see Eq. (2)).

To give an idea of the SL contamination in absolute terms, using the $\log N-\log S$ calculated by Moretti et al. (2003), we find that for each XRT image we expect a contaminating flux of $2.7 \pm 0.1 \times 10^{-13} \mathrm{erg} \mathrm{s}^{-1}$ diffuse over the 200 pixel central circle in the $1.5-7 \mathrm{keV}$ band, corresponding to a count rate of $6.3 \pm 0.2 \times 10^{-3}$ (assuming a spectral photon index of 1.4).

\section{Vignetting}

Because the CXRB is an extended source with a uniform surface brightness profile, the variation of the effective area as function of the off-axis angle, i.e. the vignetting, must be accounted for. For each energy the vignetting correction can be analytically described by a polynomial function (Tagliaferri et al. 2004). Therefore, first, we calculate the vignetting correction as function of the off-axis angle using the standard calibration (CALDB coefficients SwXvign $20010101 \mathrm{v} 001$. fits). Then, the total vignetting factor $\left(f_{\text {vign }}(E)\right.$ in Eq. $\left.(1)\right)$ is given by the integration of this function over the 200 pixels radius circular region. In the $1.5-7 \mathrm{keV}$ energy band the integrated vignetting correction ranges from $6 \%$ to $14 \%$. To estimate the vignetting calibration accuracy, which is a relevant factor in the uncertainty calculation of our measurement, we consider all the point-like sources (1945 sources) detected in our survey, excluding GRB afterglows. We calculate the median count rate of these sources in different off-axis angle bins. Assuming that the source populations detected at different off-axis angles coincide (once we eliminate GRB afterglows) and having the necessary statistics to make cosmic variance negligible, the ratio between the median count-rate on- and off-axis give us the vignetting coefficient. We repeat this operation for different energy bands and we find that

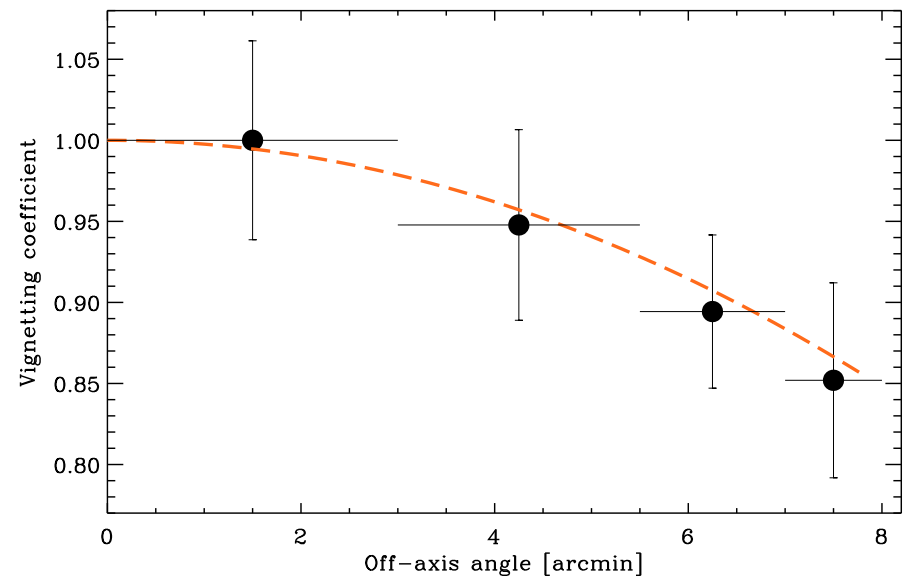

Fig. 6. Variations of the effective area as function of the off-axis angle, usually called vignetting. The dashed line represents the standard calibration function, while the black points are the values we find for the $2-3 \mathrm{keV}$ energy band.

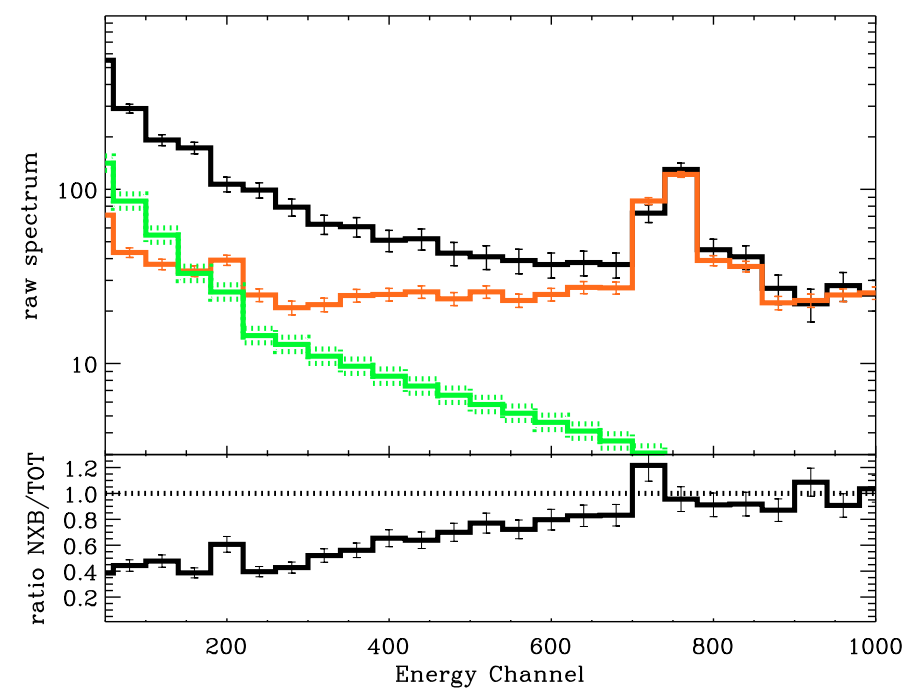

Fig. 7. The different components of the signal registered during an observation with no bright source present in the field of view. The black line is the whole signal $\left(S_{\text {tot }}\right)$ registered during the $35 \mathrm{ks}$ observation of the afterglow of GRB080319C. Data have been reduced and filtered as explained in Sect. 3 The red line is the NXB component (NES data). The green line is the expected SL contamination. In the lower panel the ratio between the total NXB (instrumental+particle induced + SL) and the $S_{\text {tot }}$ is plotted. The XRT energy channel are $0.1 \mathrm{keV}$ wide.

the standard vignetting calibration is accurate at the level of a few percent (Fig. 6).

\section{Spectral analysis}

The XRT nominal energy band is from 0.3 to $10 \mathrm{keV}$. The fraction of $S_{\text {tot }}$ due to non-cosmic background (NXB+SL) during a typical XRT observation (without any bright source in the FOV) depends on energy and is shown in Fig. 7. In the energy range between 1 and $6 \mathrm{keV}$ the NXB contributes $\sim 50 \%$ of the total signal registered during the observation. As a comparison, in the XMM-Newton data the cosmic fraction of the total signal is $20 \%$ (De Luca \& Molendi 2004). Above $7 \mathrm{keV}$ the NXB and, in particular, the particle induced background becomes dominant, due to the presence of the $\mathrm{Ni}\left(\mathrm{K}_{\alpha}\right.$ and $\mathrm{K}_{\beta}$ at 7.478 and $\left.8.265 \mathrm{keV}\right)$ and $\mathrm{Au}\left(\mathrm{L}_{\alpha}\right.$ at $\left.9.713 \mathrm{keV}\right)$ fluorescence lines. We only consider 
Table 2. The best fit results for the three different models we use. Among brackets are reported the statistical contribution to the total error budget.

\begin{tabular}{|c|c|c|c|c|c|}
\hline Model & Range [keV] & Norm. $\left[\mathrm{keV}^{-1} \mathrm{~cm}^{-2} \mathrm{~s}^{-1} \mathrm{deg}^{-2}\right]$ & Ph. ind. $(\Gamma)$ & Flux 2-10 $\left[\mathrm{erg} \mathrm{cm}^{-2} \mathrm{~s}^{-1} \mathrm{deg}^{-2}\right]$ & $\chi^{2}$ (d.o.f.) \\
\hline$\overline{\mathrm{PL}}$ & $1.5-7$ & $3.69_{-0.20(0.18)}^{+0.20(0.18)} \times 10^{-3}$ & $1.47_{-0.07(0.06)}^{+0.07(0.06)}$ & $2.18_{-0.13(0.02)}^{+0.12(0.02)} \times 10^{-11}$ & $209.1(175)$ \\
\hline $\mathrm{CPL}$ & $1.5-7$ & $3.70_{-0.20(0.18)}^{+0.20(0.18)} \times 10^{-3}$ & $1.41_{-0.06(0.06)}^{+0.06(0.06)}$ & $2.13_{-0.13(0.02)}^{+0.13(0.02)} \times 10^{-11}$ & $209.7(175)$ \\
\hline Model & Range [keV] & Norm. $\left[\mathrm{keV} \mathrm{cm} \mathrm{cm}^{-2} \mathrm{~s}^{-1} \mathrm{sr}^{-2}\right]$ & $\Gamma_{1}, \Gamma_{2}, \mathrm{E}_{\mathrm{B}}$ & Flux 2-10 keV $\left[\mathrm{erg} \mathrm{cm}^{-2} \mathrm{~s}^{-1} \mathrm{deg}^{-2}\right]$ & $\chi^{2}$ (d.o.f.) \\
\hline 2SJPL & $1.5-200$ & $0.109_{-0.003}^{+0.003} \mathrm{keV}$ & $1.40_{-0.02}^{+0.02}, 2.88_{-0.05}^{+0.04}, 29.0_{-0.5}^{+0.5}$ & $2.21_{-0.07}^{+0.07} \times 10^{-11}$ & $200.5(193)$ \\
\hline
\end{tabular}

the energy band between 1.5 and $7 \mathrm{keV}$, excluding the data with energy less than $1.5 \mathrm{keV}$ because the Galaxy contribution is not negligible (Kushino et al. 2002; Hickox \& Markevitch 2006).

We account for the vignetting by modifying the nominal ancillary response file (ARF, swxpcoto12s0(6)20010101v010.arf) by the $f_{\text {vign }}(E)$ factor (see Sect. 6). We modify the ARF file also to account for the SL contamination according to Eq. (2) with the results reported in Sect. 5. Finally, we calculate the contamination of the GRB afterglow residuals outside the 30 pixel radius using the analytical PSF model Moretti et al. (2005). The GRB residual signal is energy dependent and contributes a maximum of $1.1 \%$ below $2 \mathrm{keV}$, becoming negligible above $3 \mathrm{keV}$. We account for this contamination applying another small energy dependent correction to the ARF file. To summarize, for each energy $E$ we modify the nominal ARF according:

$\operatorname{ARF}^{\prime}(E)=\operatorname{ARF}(E) \times\left(f_{\text {vign }}(E)+f_{\mathrm{SL}}(E)\right) \times\left(1-\operatorname{GRB}_{\text {res }}(E)\right)$.

In order to account for the CCD defects and the excluded 30 pixel radius circular region around the GRB position we create an exposure map for each observation. The overall correction, weighted for the exposure time of the single observation, correspond to $6.3 \%$ for the sky exposure and $3.2 \%$ for the background. We correct this by modifying the BACKSCAL keyword in the spectrum (PHA) files. For the background file we consider the NES regions that provide better statistics than the SC observations (Sect. 4). The four NES regions are not homogeneous due to the spatial gradient in the NXB. Nevertheless the symmetry of the geometry allows us to use this dataset without any correction. We perform the stacked spectral analysis merging the 126 event files, re-sorting the events and the GTIs, and extracting the spectrum from the 200 pixel radius central circle in detector coordinates. To fit the data and calculate the fluxes we use XSPEC(v12.4).

To account for the systematic uncertainties in the NXB (Sect. 4), vignetting factor $\left(f_{\text {vign }}\right.$, Sect. 6$)$ and SL contamination $\left(f_{\mathrm{SL}}\right.$, Sect. 5) measurement we produce a large number $(10000)$ of simulated datasets, randomly varying the NXB normalization, the $f_{\text {vign }}$ and $f_{\mathrm{SL}}$, according to the appropriate Gaussian distributions. For the NXB normalization we use the mean standard deviation that we observe for the $\mathrm{Ni}$ line fluxes in our sample (lower panel of Fig. 5). For the $f_{\text {vign }}$ and $f_{\mathrm{SL}}$ we conservatively use a standard deviation equal to $5 \%$ which slightly exceeds the estimated errors.

We neglect the Galactic contribution (absorption and emission) and fit our data by means of a simple power law, obtaining the numbers reported in Table 2 and shown in Fig. 8. For analogy with previous works in the literature (Gruber et al. 1999; Frontera et al. 2007), we also fit our data with a cut-off power law (CPL) with the energy break fixed to $41.13 \mathrm{keV}$. Both the models provide a good description of our data in the $1.5-7 \mathrm{keV}$ energy interval.

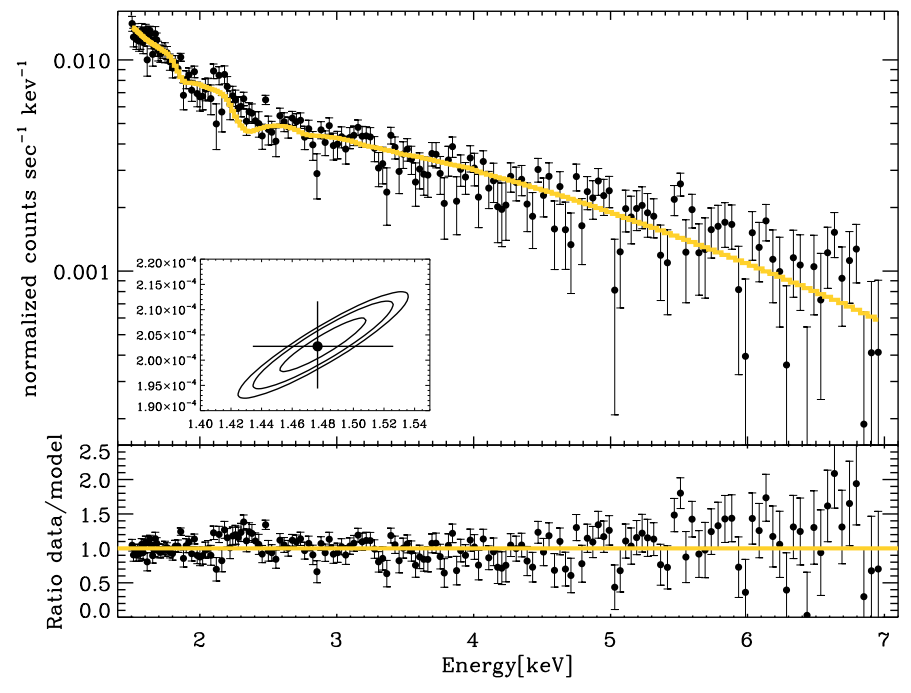

Fig. 8. CXRB spectrum and the power law best fit. Best fit values are reported in Table 2.

Finally we combine our data with the Swift-BAT CXRB measurement performed in the energy band 20-150 keV (Ajello et al. 2008) and we fit the joined energy distributions with two smoothly joined power laws (2SJPL) of the form

$E^{2} \cdot \frac{\mathrm{d} N}{\mathrm{~d} E}=\frac{C \cdot E^{2}}{\left(E / E_{\mathrm{B}}\right)^{\Gamma_{1}}+\left(E / E_{\mathrm{B}}\right)^{\Gamma_{2}}}\left[\mathrm{keV} \mathrm{cm}^{-2} \mathrm{~s}^{-1} \mathrm{deg}^{-2}\right]$

This is the same model Ajello et al. (2008) uses to fit a large collection of CXRB measurements, together with the Swift-BAT new measurement. We note that, because a specific response matrix has been produced in the Swift-BAT measurement, the cross-calibration factor for point-like sources (Godet et al. 2009) cannot be applied here.

Our best fit values are reported in Table 2. The peak of the energy distribution $E_{\text {peak }}$ is given by

$E_{\text {peak }}=E_{\mathrm{B}} \cdot\left(\frac{2-\Gamma_{2}}{\Gamma_{1}-2}\right)^{\frac{1}{\Gamma_{1}-\Gamma_{2}}}=22.4 \pm 0.4 \mathrm{keV}$

\subsection{Cosmic variance}

To study the variance of our sample we consider the 113 observations with durations longer than $10 \mathrm{ks}$ for which the spectral parameters and the flux of the CXRB can be calculated with an acceptable accuracy $\left(\sigma_{\mathrm{s}} \sim 15 \%\right)$. We find that the flux distribution is well described by a Gaussian with a standard deviation of $\sigma_{\mathrm{o}}=20.8 \% \pm 2.4$ (upper panel of Fig. 9). The maximum CXRB flux value in our sample, at $\sim 5 \sigma_{\mathrm{o}}$ from the mean, is observed in the field of GRB 071028B where the galaxy cluster Abell S1136 is present. This is not surprising, given the fact that 

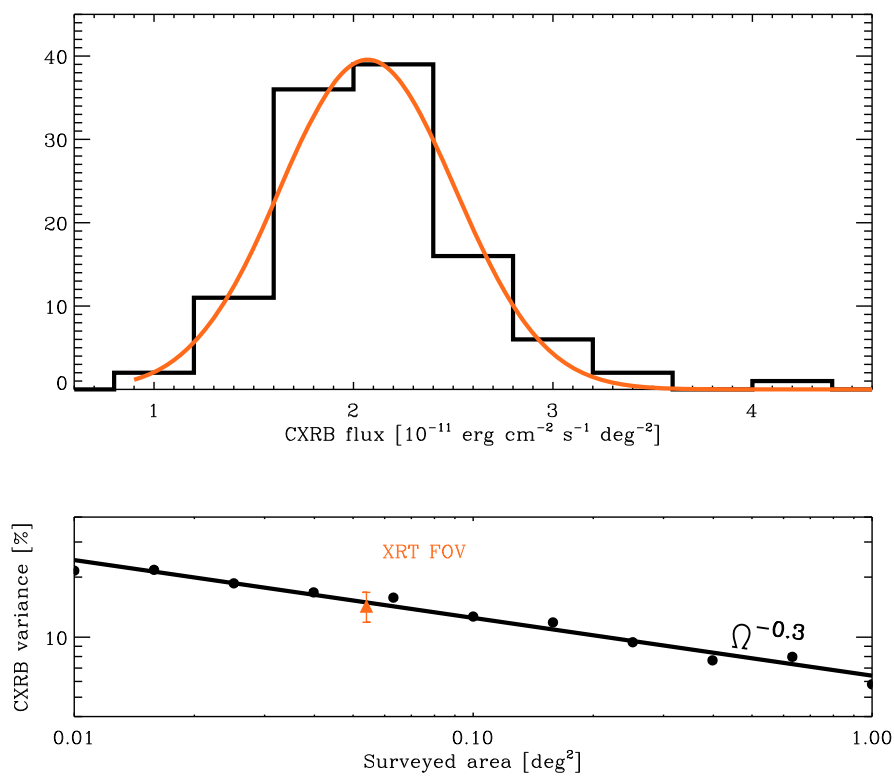

Fig. 9. Upper panel: the distribution of the CXRB flux measurements from the 113 observations with effective durations longer than $10 \mathrm{ks}$ (black line), together with the best Gaussian fit (red line). The scatter in the measurements is the sum of the statistical error $(\sim 15 \%)$ and cosmic variance. Lower panel: the comparison between CXRB variance expected (continuous line and small circles) with the observed one, corrected for the statistical contribution (triangle).

Abell clusters are $\sim 5000$ distributed over $\sim 27000 \mathrm{deg}^{2}$ of sky meaning that 1-2 Abell clusters are expected in our survey.

The variance we observe in the flux distribution is contributed by both by the statistical error $\left(\sigma_{\mathrm{s}}\right)$ and cosmic variance $\left(\sigma_{\mathrm{c}}\right)$. We find that the latter is consistent with the CXRB variance expected for the area surveyed by a single observation if we assume that the CXRB is entirely produced by point sources. In fact, it can be shown that, if we assume that the source fluxes are distributed as the classical $F^{-3 / 2} \log N-\log S$, the cosmic variance scales with the surveyed area as $\Omega^{-0.5}$, (Revnivtsev et al. 2008). Assuming the $\log N-\log S$ calculated by Moretti et al. (2003), which is flatter at low energies and generating 1000 random samples with different dimensions (ranging from 0.01 to 1 square degree of sky), in the flux range $10^{-16}-10^{-10} \mathrm{erg} \mathrm{cm}^{-2} \mathrm{~s}^{-1}$, we find that a more realistic value is $\Omega^{-0.3}$, as shown in the lower panel of Fig. 9. We find that, with $0.054 \mathrm{deg}^{2}$ XRT field, we expect a variance of $15.1 \%$. This is very close to the one we observe in our sample, once we account also for the contribution of the statistical error: $\sigma_{\mathrm{c}}^{2}=\sigma_{\mathrm{o}}^{2}-\sigma_{\mathrm{s}}^{2}=14.3 \pm 1.8 \%$. We note also that the extrapolation of our simulations to the total surveyed area $\left(\sim 7 \mathrm{deg}^{2}\right)$ tells us that the stacked analysis uncertainty due to cosmic variance is negligible.

\subsection{Data check}

We check our data against any bias due to the time of the observation, CCD temperature, and Galactic latitude. As explained in Sect. 4 we find a slight dependence of the NXB on the time of the observations. However, as the NXB dependence is slight and the NXB contribution is minor the 113 CXRB flux measurements do not have any significant correlation with the observation time (see lower panel of Fig. 10). As already said (Sect. 3) the XRT CCD temperature is variable due to the fact that it is only passively controlled. Because dark current and hot pixels
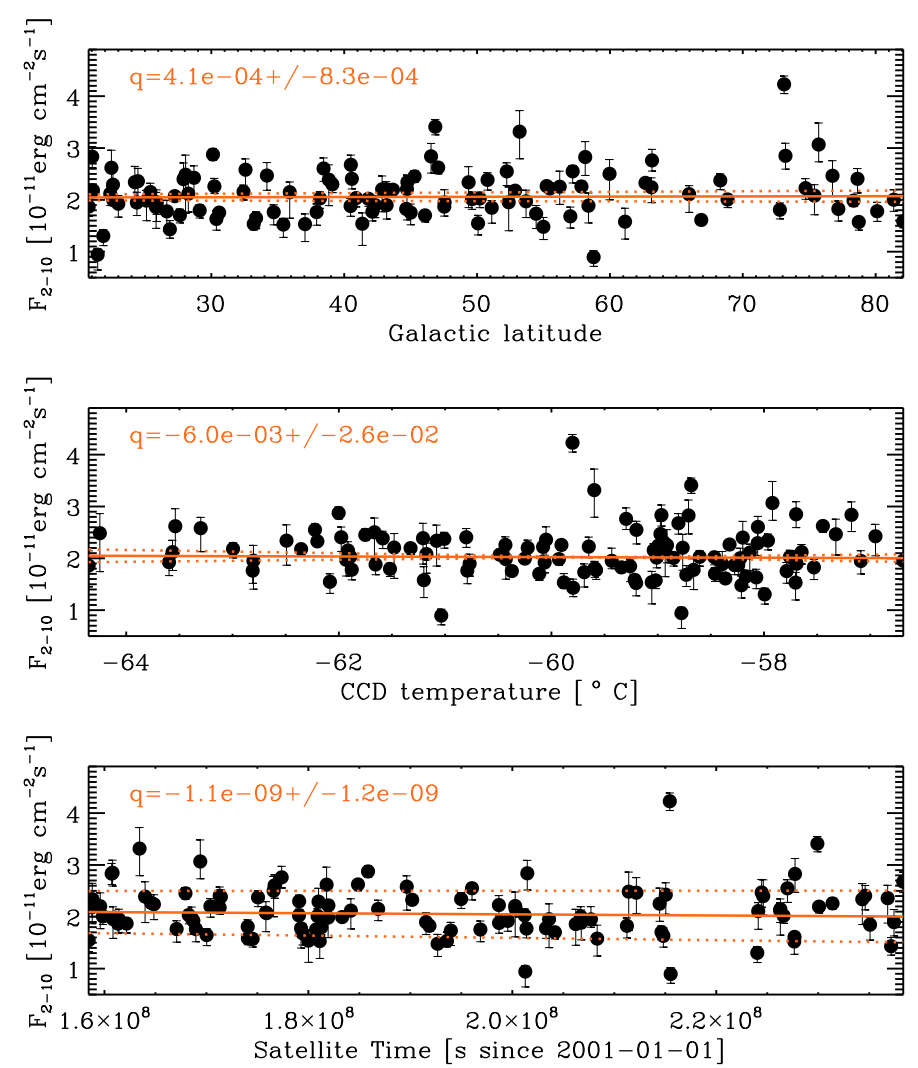

Fig. 10. CXRB measures plotted against time, Galactic latitude and CCD temperature. Here the plotted error are only the statistical ones. We fit all the three datasets with a straight line $(y=q x+a)$, finding that they are consistent with a constant $(q=0)$ at the level of $1 \sigma$.

are temperature dependent, we also plot the 113 flux measurements against the average temperature of the observations (see central panel of Fig. 10). Finally we check the flux measurements against Galactic latitude to exclude any significant contribution from our galaxy to the XRT measurements (upper panel of Fig. 10). For all the three datasets we find that the best linear fit is consistent with a constant at the level of $1 \sigma$.

\section{Discussion}

As mentioned in the Introduction, the CXRB spectrum normalization is still a debated issue.

The Swift-XRT measurement, we present here, is very close to XMM-Newton (Table 1). This is not unexpected as the SwiftXRT effective area calibration has been slightly modified to match XMM-Newton by means of simultaneous observations ${ }^{3}$. For what concerns cross-calibration, Swift-XRT measures fluxes 5-10\% lower than RXTE-PCA during simultaneous observations of 3c273 (Godet et al. 2009). Cross-calibration observations of 1E 0102.2-7219, the brightest supernova remnant in the Small Magellanic Cloud, recently showed that ChandraACIS, XMM-Newton-MOS, Suzaku-XIS and Swift-XRT agree to within $\pm 10 \%$ for all instruments (Plucinsky et al. 2008). Therefore, the differences with HEAO1 and RXTE-PCA measurements cannot be entirely explained by the absoulte calibration differences, as already pointed out by Frontera et al. (2007).

\footnotetext{
3 SWIFT-XRT-CALDB-09-V11 available at http://heasarc. gsfc.nasa.gov/docs/heasarc/caldb/swift/docs/xrt/index . html
} 


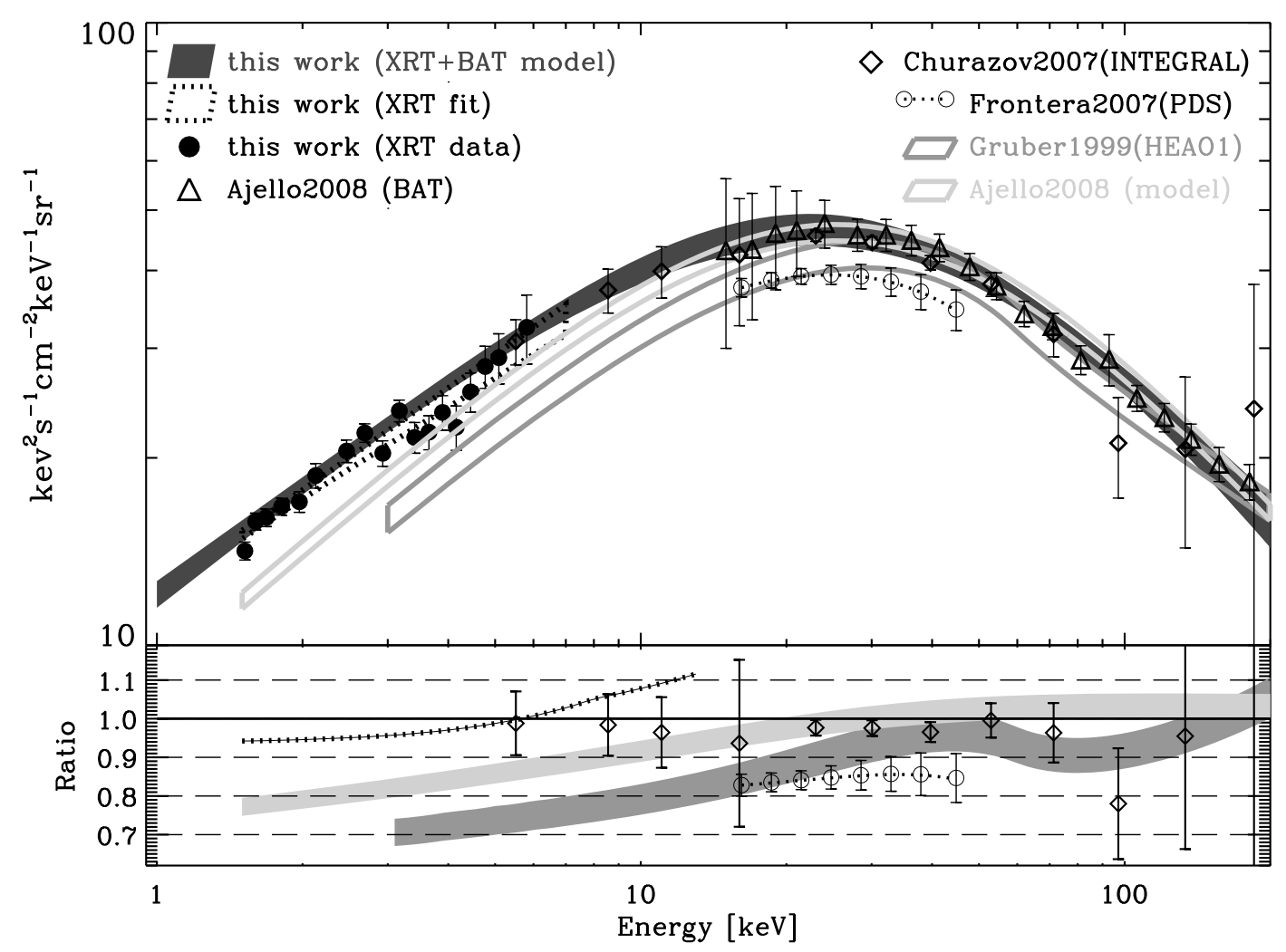

Fig. 11. Upper panel: a compilation of flux measurements both in the soft and hard energy bands. For the clearness of the plot, not all the soft energy measurements reported in Table 1 are shown here. Because Gruber et al. (1999) do not report the uncertainties in the best fit values, we use a fiducial 5\% error for G99 model. Lower panel: ratio of the flux measurements plotted in the upper panel with our joined XRT+BAT fit. Colors are the same of upper panel. For comparison, with the dotted line we plot (only in the bottom panel) the ad-hoc model Worsley et al. (2005) used to calculate the resolved fraction.

In the region of the CXRB peak $(\sim 30 \mathrm{keV})$ all the measurements show a acceptable agreement $(\sim 10 \%)$, in the soft band the XRT measurement confirms Revnivtsev et al. (2005) conclusions: in the 2-10 keV band narrow-field focusing telescopes measure CXRB values which are significantly higher than the ones found by wide-field not focusing telescopes. However, the XRT data, although inconsistently higher than the G99 model, smoothly join the higher energy data as we show by the good fit to the XRT and BAT.

Below $60 \mathrm{keV}$, the G99 model consists in a CPL with $\Gamma=$ 1.29 and energy break 41.13 (note that no uncertainties are reported in the Gruber et al. 1999 paper). As shown in Fig. 11, the differences from the G99 model range from $30 \%$ below $10 \mathrm{keV}$ down to $5-10 \%$ in the region of the CXRB energy peak. This is due to the fact that the slope of the soft part in our best fit is steeper (1.41 instead of 1.29) and the peak of the spectrum is much softer ( $22 \mathrm{keV}$ instead of $29 \mathrm{keV})$. As previously discussed, Ajello et al. (2008) uses a 2SJPL to fit Swift-BAT data together with a large collection of different CXRB measurements down to $2 \mathrm{keV}$. In comparison to this model we find that the soft energy slope is significantly softer $(1.41 \pm 0.02$ versus $1.32 \pm 0.02$ ), while the high energy slope, the energy break and the normalization are consistent. Interestingly, our model has the same CPL shape, with energy break at $41.13 \mathrm{keV}$ and photon index 1.4, that provides the best fit to SAX-PDS data (Frontera et al. 2007) in the $20-50 \mathrm{keV}$ band, albeit with a significant difference in normalization. Finally we also note that XRT data and our model are well consistent with the INTEGRAL measurement (Churazov et al. 2007) all over the considered energy band.
In summary, breaking the paradigm that G99 spectrum has the correct shape shows that CXRB data can be analytically described by a 2SJPL with the values reported Table 2 and a peak in the energy distribution at $22.9 \pm 0.4 \mathrm{keV}$. In the $1.5-50 \mathrm{keV}$ range, this function is very similar to a CPL with the energy break of $41.13 \mathrm{keV}$ and photon index of 1.4 .

We note that the 2-10 keV CXRB flux measurement directly affects the calculation of the CXRB resolved fraction. Moretti et al. (2003), combining shallow and deep surveys and integrating the source number counts, estimate that the resolved fraction of $2-10 \mathrm{keV}$ CXRB is $87 \pm 6 \%$. Worsley et al. (2005) refined this calculation finding that the resolved fraction ranges from $80 \%$ in the $2-4 \mathrm{keV}$ band to $\lesssim 60 \%$ for energies higher than $6 \mathrm{keV}$. The main reason for the inconsistency between the two results is the value of the CXRB total flux. Moretti et al. (2003) used an average of a sample of CXRB measurements, yielding a value of $2.02 \pm 0.11 \times 10^{-11} \mathrm{erg} \mathrm{cm}^{-2} \mathrm{~s}^{-1} \mathrm{deg}^{-2}$ which is $10 \%$ less than the present measurement. As already mentioned, Worsley et al. (2005) used an ad hoc model, combining the XMM-Newton measurement with a re-normalized G99 model. As shown in the Fig. 11 (bottom panel) this model, although not motivated from an observational point of view, is not very far from our best fit. If we assume the present measurement for the CXRB and integrate the $\log N-\log S$ of Moretti et al. (2003), we find a result for the CXRB resolved fraction which is $79 \pm 6 \%$ in the $2-10 \mathrm{keV}$ band, in very good agreement with the average value quoted by Worsley et al. (2005). The values relative to the single narrow bands at higher energies, on the other hand, should be slightly corrected, applying our CXRB value. 
The $\log N-\log S$ extrapolation at very low fluxes $\left(10^{-17} \mathrm{erg} \mathrm{cm}^{-2} \mathrm{~s}^{-1} \mathrm{deg}^{-2}\right.$, a factor 20 lower than the faintest Chandra deep field sources) cannot account for all the CXRB. This implies that a not negligible fraction of the CXRB is supposed to be produced by non detected sources. Worsley et al. (2006) and Hickox \& Markevitch (2007) correlate almost the entire CXRB unresolved fraction to optical/IR detected galaxies in the Chandra deep fields. These are star-forming galaxies which are expected to overwhelm the number of AGNs at very low fluxes (Ranalli et al. 2003; Bauer et al. 2004), absorbed AGN (Treister \& Urry 2005; Gilli et al. 2007) which are supposed to be the main component at higher energies and with a small contribution from very high redshift $(z>6)$ quasars.

\section{Conclusion}

We use the Swift-XRT archival dataset to determine the flux and spectrum of the CXRB. This has two main advantages. The first one is the observational strategy which provides us with a truly random sampling of the X-ray sky, not correlated with previously known sources. The second is the low level of the NXB background, which allows measurement of the CXRB with high accuracy. Similar to other focusing telescopes, we find that CXRB flux is significantly higher than HEAO1/G99 model. Nevertheless combining our dataset with Swift-BAT data, we show that we can describe the CXRB spectrum with a simple model (two smoothly joined power laws) over a wide energy band. The model we propose is much more observationally motivated than the ones recently used in the literature for population synthesis models and for the CXRB resolved fraction calculation. Using the present CXRB measurement we calculate that the resolved fraction in the $2-10 \mathrm{keV}$ energy band is $79 \pm 6 \%$.

Acknowledgements. This work is supported at OAB-INAF by ASI grant I/011/07/0, at PSU by NASA contract NAS5-00136. A.A., A.B., O.G. and J.O. acknowledge STFC funding. This research has made use of NASA's Astrophysics Data System Service.

\section{References}

Ajello, M., Greiner, J., Sato, G., et al. 2008, ApJ, 689, 666

Ballantyne, D. R., \& Papovich, C. 2007, ApJ, 660, 988

Barthelmy, S. D., Barbier, L. M., Cummings, J. R., et al. 2005, Space Sci. Rev., 120,143

Bauer, F. E., Alexander, D. M., Brandt, W. N., et al. 2004, AJ, 128, 2048

Brandt, W. N., \& Hasinger, G. 2005, ARA\&A, 43, 827

Burrows, D. N., Hill, J. E., Nousek, J. A., et al. 2005, Space Sci. Rev., 120, 165

Cen, R., \& Ostriker, J. P. 1999, ApJ, 514, 1

Churazov, E., Sunyaev, R., Revnivtsev, M., et al. 2007, A\&A, 467, 529

Citterio, O., Conconi, P., Ghigo, M., et al. 1994, in SPIE Conf. Ser. 2279, ed.

R. B. Hoover, \& A. B. Walker, 480
De Luca, A., \& Molendi, S. 2004, A\&A, 419, 837

Frontera, F., Orlandini, M., Landi, R., et al. 2007, ApJ, 666, 86

Galeazzi, M., Gupta, A., Covey, K., \& Ursino, E. 2007, ApJ, 658, 1081

Gastaldello, F., Buote, D. A., Humphrey, P. J., et al. 2007, ApJ, 669, 158

Gehrels, N., Chincarini, G., Giommi, P., et al. 2004, ApJ, 611, 1005

Gendreau, K. C., Mushotzky, R., Fabian, A. C., et al. 1995, PASJ, 47, L5

Giacconi, R., Gursky, H., Paolini, F. R., \& Rossi, B. B. 1962, Phys. Rev. Lett., 9, 439

Giacconi, R., \& Zamorani, G. 1987, ApJ, 313, 20

Gilli, R., Comastri, A., \& Hasinger, G. 2007, A\&A, 463, 79

Godet, O., Beardmore, A. P., Abbey, A. F., et al. 2009, A\&A, [arXiv: 0811.4246]

Gruber, D. E., Matteson, J. L., Peterson, L. E., \& Jung, G. V. 1999, ApJ, 520, 124

Hickox, R. C., \& Markevitch, M. 2006, ApJ, 645, 95

Hickox, R. C., \& Markevitch, M. 2007, ApJ, 661, L117

Hornschemeier, A. E., Brandt, W. N., Garmire, G. P., et al. 2000, ApJ, 541, 49

Kennea, J. A., Burrows, D. N., Wells, A., et al. 2005, in SPIE Conf. Ser. 5898, ed. O. H. W. Siegmund, 341

Kirsch, M. G., Briel, U. G., Burrows, D., et al. 2005, in SPIE Conf. Ser. 5898, ed. O. H. W. Siegmund, 22

Kushino, A., Ishisaki, Y., Morita, U., et al. 2002, PASJ, 54, 327

McCammon, D., Burrows, D. N., Sanders, W. T., \& Kraushaar, W. L. 1983, ApJ, 269, 107

Moretti, A., Campana, S., Lazzati, D., \& Tagliaferri, G. 2003, ApJ, 588, 696

Moretti, A., Campana, S., Mineo, T., et al. 2005, in SPIE Conf. Ser. 5898, ed. O. H. W. Siegmund, 360

Moretti, A., Perri, M., Capalbi, M., et al. 2007, in SPIE Conf. Ser. 6688, ed. S. L. O’Dell, \& G. Pareschi

Pagani, C., Morris, D. C., Racusin, J., et al. 2007, in SPIE Conf. Ser. 6686, ed. H. Siegmund

Perri, M., \& Giommi, P. 2000, A\&A, 362, L57

Plucinsky, P. P., Haberl, F., Dewey, D., et al. 2008, ArXiv e-prints, 807

Ranalli, P., Comastri, A., \& Setti, G. 2003, A\&A, 399, 39

Revnivtsev, M., Gilfanov, M., Sunyaev, R., Jahoda, K., \& Markwardt, C. 2003, A\&A, 411, 329

Revnivtsev, M., Gilfanov, M., Jahoda, K., \& Sunyaev, R. 2005, A\&A, 444, 381

Revnivtsev, M., Molkov, S., \& Sazonov, S. 2008, A\&A, 483, 425

Rhook, K. J., \& Haehnelt, M. G. 2008, MNRAS, 389, 270

Salvaterra, R., Haardt, F., \& Volonteri, M. 2007, MNRAS, 374, 761

Sazonov, S., Revnivtsev, M., Krivonos, R., Churazov, E., \& Sunyaev, R. 2007, A\&A, 462, 57

Sazonov, S., Krivonos, R., Revnivtsev, M., Churazov, E., \& Sunyaev, R. 2008, A\&A, 482, 517

Setti, G., \& Woltjer, L. 1989, A\&A, 224, L21

Shafer, R. A., \& Fabian, A. C. 1983, in Early Evolution of the Universe and its Present Structure, ed. G. O. Abell, \& G. Chincarini, IAU Symp., 104, 333

Snowden, S. L., Mushotzky, R. F., Kuntz, K. D., \& Davis, D. S. 2008, A\&A, 478,615

Sołtan, A. M. 2007, A\&A, 475, 837

Tagliaferri, G., Moretti, A., Campana, S., et al. 2004, in SPIE Conf. Ser. 5165, ed. K. A. Flanagan, \& O. H. W. Siegmund, 241

Tozzi, P., Gilli, R., Mainieri, V., et al. 2006, A\&A, 451, 457

Treister, E., \& Urry, C. M. 2005, ApJ, 630, 115

Tueller, J., Mushotzky, R. F., Barthelmy, S., et al. 2008, ApJ, 681, 113

Ueda, Y. 2007, Nature Phys., 3, 450

Ueda, Y., Akiyama, M., Ohta, K., \& Miyaji, T. 2003, ApJ, 598, 886

Vecchi, A., Molendi, S., Guainazzi, M., Fiore, F., \& Parmar, A. N. 1999, A\&A, 349, L73

Worsley, M. A., Fabian, A. C., Bauer, F. E., et al. 2005, MNRAS, 357, 1281

Worsley, M. A., Fabian, A. C., Bauer, F. E., et al. 2006, MNRAS, 368, 1735 